\title{
Investigation of the Interactions of Reaction Control Systems with Mars Science Laboratory Aeroshell
}

\author{
Erin M. Reed, Josh Codoni, James C. McDaniel \\ Department of Mechanical and Aerospace Engineering, University of Virginia \\ Charlottesville Virginia 22904 \\ Hicham Alkandry, Iain D. Boyd \\ Department of Aerospace Engineering, University of Michigan \\ Ann Arbor Michigan 48109
}

\begin{abstract}
Interactions between the Reaction Control System (RCS) jets and the bow shock from the aeroshell of a Mars Science Lab (MSL) model are investigated. Images are obtained experimentally at the University of Virginia using a low-density, hypersonic wind tunnel with the Planar Laser Induced Iodine Fluorescence technique. The models are .44\% MSL aeroshells fitted with $0.5 \mathrm{~mm}$ RCS orifices to simulate Reaction Control Systems in both parallel and transverse jet directions relative to the aeroshells. Experiments are conducted at Mach 12 in the underexpanded jet freestream flowfield with sonic RCS jets. Images for both transverse and parallel jets are obtained for nozzle-thrust coefficients ranging from 0 to 3 . It is found that there is much interaction between the aeroshell bow shock and the RCS jet for the transverse jet cases; however, there was not much interaction between the parallel jet and the bow shock on the aeroshell. Results from a nozzle-thrust coefficient of 0.5 were compared to numerical simulations for similar conditions obtained using CFD at the University of Michigan. It is found that there is good agreement in flowfield density between the experimental and numerical results in the jet core of the RCS but greater differences near the jet boundaries.
\end{abstract}

\section{Introduction}

To date, six vehicles have successfully landed on Martian soil. This is no easy feat considering the challenges for entry, descent and landing. With a much thinner atmosphere than Earth, the Mars atmosphere proves a challenge to slow vehicles to terminal velocity at high enough altitudes for maneuvering to a safe landing location $[1,2]$. The landscape of Mars itself also proves to be a challenge with rocks and craters that could damage the landing vehicle [1]. As a result, large landing footprints on the order of 100's of km have been chosen in relatively rock-free regions of Mars at low altitudes [1]. The Mars Science Laboratory, which will prove heavier and larger than any other vehicle to navigate to Mars, is required to make great strides in improving landing accuracy with a $10 \mathrm{~km}$ radius landing footprint $[3,4,5,6]$. Thus, MSL will have access to regions of scientific interest previously unavailable due to its risk to the mission [6,7]. While previous missions have landed at sites below -1 km Mars Orbiter Laser Altimeter (MOLA), it is anticipated that MSL will land at a site $+2 \mathrm{~km}$ MOLA $[1,4]$. The improved accuracy of MSL will prove an advantage for subsequent robotic missions to Mars and critical for future manned missions to Mars which will require footprints on the order of 10 's of meters $[1,7]$.

In order to obtain these high accuracy landings - precision landing for MSL and pinpoint landing in the future - there is the need to navigate the vehicle to allow the 
vehicle to be in position for a safe landing [4]. This is done through the effective use of Reaction Control System (RCS) jets at altitudes where normal control surfaces prove ineffectual. RCS jets utilize yaw and roll commands in order to orient the lift vector of the entry vehicle to provide precision landings. There is still much that is not fully understood about the aerodynamic interactions of the RCS jet on the aeroshell. For instance, one current concern with RCS jets is that heating at the critical shoulder of the MSL is actually augmented when firing the RCS jets [8]. Thus, it is important to better understand the aerodynamic interactions between a RCS jet and an aeroshell in order to improve this technology so that it can be reliably used in manned missions.

The objective of this investigation is to increase fundamental understanding of the aerodynamic interactions of the RCS jets with the MSL aeroshell in a hypersonic freestream through both experimental and computational methods completed at the University of Virginia and the University of Michigan. Using a method called Planar Laser-Induced Iodine Fluorescence, time-averaged, spatially-resolved images of an MSL model fitted with parallel and transverse RCS jets are taken to obtain a qualitative understanding about the interactions between the jet and the aeroshell, at thrust coefficents ranging from 0.5 to 3 . Experimental results are then compared to computations performed at the University of Michigan for the parallel jet case at a thrust coefficient of 0.5 .

\section{Background}

\section{A. PLIIF Experimental Method (University of Virginia)}

\section{PLIIF:}

Planar Laser Induced Iodine Fluorescence (PLIIF) is a method developed and used at the University of Virginia for the past 26 years [11,12,13,14]. PLIIF is a nonintrusive, spatially resolved optical diagnostic method. In this method, the flow is seeded with iodine molecules. Broadband excitation from a $514.5 \mathrm{~nm}$ argon ion laser is collimated into a laser sheet and directed through the flow. The iodine fluoresces and images are taken with a CCD camera perpendicular to the laser sheet, which allows for a planar resolution of the flow. This set-up can be seen in Figures 1 and 2. Figure 1 shows an external view of the wind tunnel set up with the large vacuum chamber fitted with optical windows, which allow visual access into the wind tunnel. Also seen in this image are the optical rails that hold optics for collimating and directing the laser sheet into the wind tunnel. Figure 2 gives an internal view of the set up. As shown, the laser sheet is directed through one optical window (circle in figure) while the camera is focused through another optical window perpendicular to the direction of the laser sheet [13]. With the broadband laser images in the work reported herein, qualitative understanding about the flow can be obtained. Quantitative data can also be extracted using this method; velocity, temperature, pressure and mole fraction mapping of the flow field can all be obtained $[11,12,13,14]$. This method is advantageous over other methods because of its ability to capture signal in both continuum and rarefied regions of the flow. Unlike Schlieren and Rayleigh scattering methods, the PLIIF method can still produce high- quality images and results even in low-density regions of the flow [11]. Images are 
obtained using an Andor iKon-L CCD camera with a 2kx2k CCD and cooled using a liquid Koolance system. The Andor camera system produces 16 bit images. Images are taken at different exposure times ranging from 4 to 17 seconds. The images produced are thus spatially resolved and time-averaged.

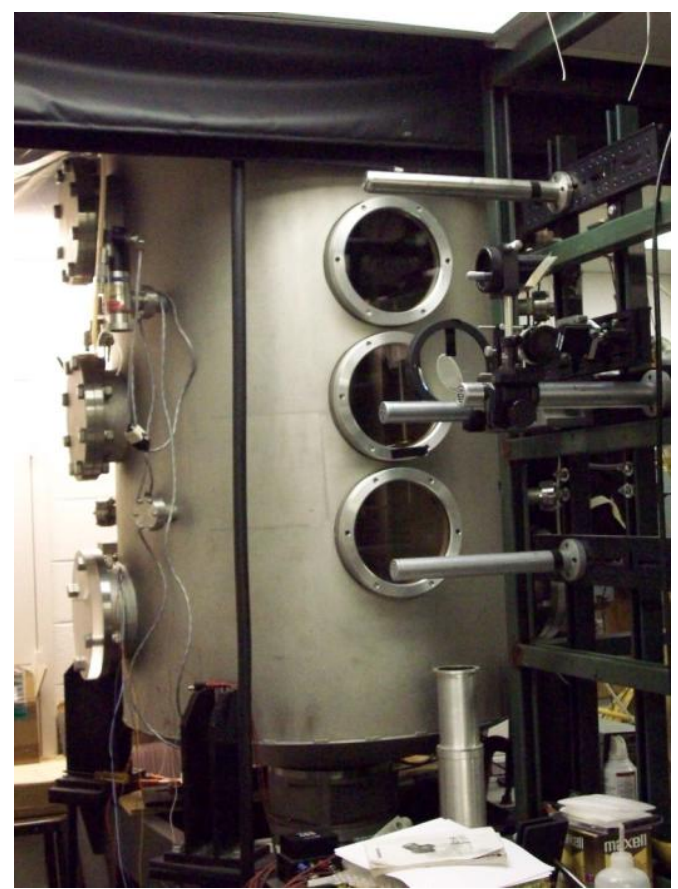

Figure 1- Image of Wind Tunnel Set Up

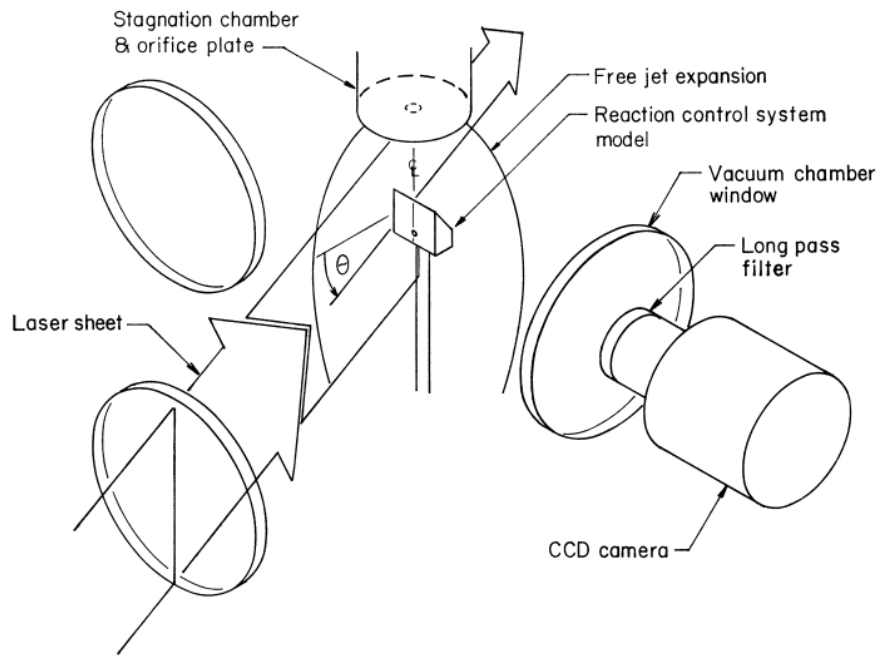

Figure 2- PLIIF Set Up

\section{Wind Tunnel:}

The wind tunnel for this experiment is a low density, hypersonic wind tunnel which uses a freely expanding jet through an orifice [9]. As the jet freely expands, it creates a barrel shock, inside which the velocity of the flowfield ranges from sonic at the orifice up to Mach 16 at the Mach disk, where the Knudsen number approaches 1. Inside this barrel shock, a model can be placed at a calibrated point which allows for testing at a specific Mach number. The Mach numbers for the various distances from the orifice have been calculated using the work done by Ashkenas and Sherman based on work with freely expanding jets [10]. Equation 1 gives the Mach number versus distance along the freejet centerline where $\mathrm{x}$ is the distance from the orifice, normalized by the orifice diameter, $\mathrm{D}, \mathrm{M}$ is Mach number, and constants $\mathrm{A}, \gamma$, and $\mathrm{x}_{0} / \mathrm{D}$ are $3.65,1.4$, and 0.075 respectively for nitrogen $\left(\mathrm{N}_{2}\right)$ gas [10].

$$
M=A\left(\frac{x-x_{0}}{D}\right)^{\gamma-1}-\frac{\frac{1}{2}\left(\frac{\gamma+1}{\gamma-1}\right)}{A\left(\frac{x-x_{0}}{D}\right)^{\gamma-1}}
$$

The visualizations for this work are conducted at Mach 12. This Mach number was chosen because the bow shock generated by the blunt body of the MSL model 
interacts with the freestream Mach disk/ barrel shock system. Working at a higher Mach number, or further downstream from the orifice, allows this interaction to be minimized. A vacuum for the wind tunnel is achieved by the use of three pumps - a Stokes MicroVac Pump, a Roots Rotary Vane Booster Pump and a Roots Rotary Vane High Pressure Pump. These pumps can achieve back pressures approximately 400 mTorr at a freejet total pressure of 1.8 atm $[9,12]$.

\section{Model Geometry:}

Two MSL/RCS model configurations are examined in this investigation. The models are $2 \mathrm{~cm}$ ( $0.8 \mathrm{in})$ in diameter or $0.44 \%$ MSL model aeroshell. Both models' RCS jets are placed in identical locations on the models (downstream of the shoulder of the forebody) with an exit velocity of Mach 1 (exit diameter of $0.5 \mathrm{~mm}$ ). The direction of the jet flow is different, however, with one jet exiting approximately perpendicular to the main freestream flow and the other jet exiting approximately parallel to the main jet flow as seen in Figure 3a. Both models are set at a 20 degree angle of attack inside the wind tunnel. A photograph of one RCS model is given in Figure 3b. It is anticipated that both of these configurations will yield information that is helpful for better understanding overall of RCS performance in response to yaw and roll commands.

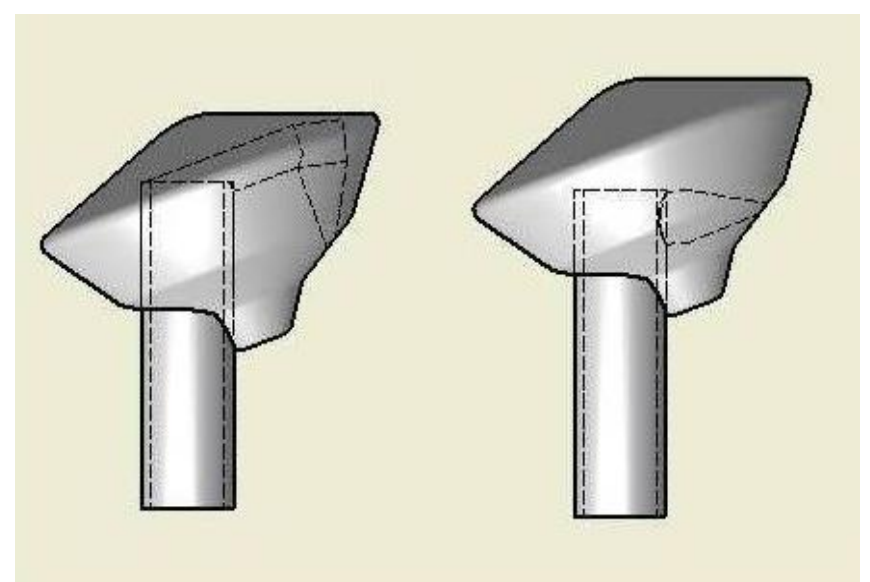

(a) Internal Geometry, Transverse Jet on left, Parallel jet on right

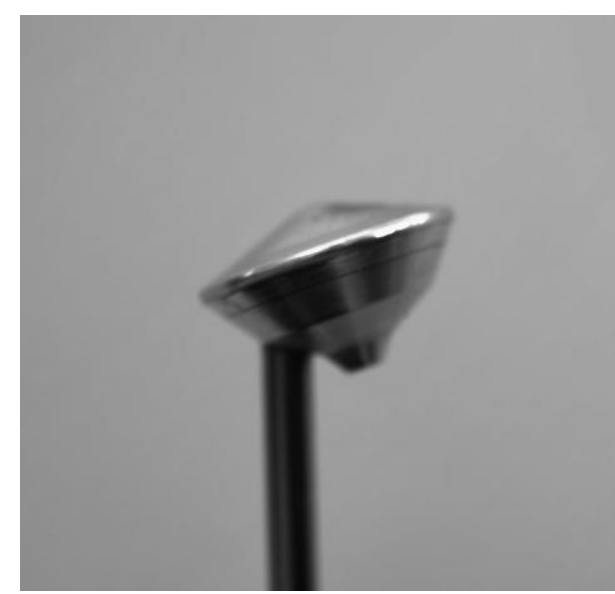

(b) External Geometry

\section{Figure 3- RCS Models}

\section{Nozzle-thrust Coefficient:}

In order to compare the experiments with previous and ongoing work done at different facilities, it is necessary to examine the experiments with reference to a non-dimensional thrust factor. Here we use the nozzle-thrust coefficient which is the ratio of the jet thrust to the freestream dynamic pressure of the main flow times the frontal area of the aeroshell $[10,11]$. The result in equation 2 was derived using isentropic relations:

$$
C_{T}=\frac{T}{q_{\infty} S}=\frac{\dot{m} V_{e}+\left(p_{e}-p_{\infty}\right) A_{e}}{q_{\infty} S}=1.810 \frac{\left(1+0.2 M_{\infty}^{2}\right)^{3.5}}{M_{\infty}^{2}}\left(\frac{A_{e}}{S}\right) \frac{p_{o, J}}{p_{o, T}}
$$


Based on this equation, and also on nozzle-thrust coefficients previously used, it was decided to run the experiment with thrust coefficients from 0 to 3 in increments of 0.5 [15]. It should be noted that these nozzle thrust coefficient values do not represent those that would be used by the MSL RCS jets. Instead they were chosen for comparison with studies previously performed as well as comparison with CFD. For thrust coefficients of 1,2 and 3, the corresponding pressure ratios given by the equation at freestream Mach 10 and Mach 12 flow are given in Table 1 below. As shown in the table, the pressure ratios for Mach 10 conditions are higher, up to almost 3 times greater, than what is required for thrust conditions at Mach 12 conditions. Thus, the experiment was conducted with a freestream Mach number of 12, in order to provide the thrust coefficients of interest with the pressure ratio available in the experiment.

Table 1: Jet Pressure to Total Pressure for Mach 10 and Mach 12

\begin{tabular}{|c|c|c|}
\hline$C_{T}$ & $P_{0, J} / P_{0, T}(M \infty=10)$ & $P_{0, J} / P_{0, T}(M \infty=12)$ \\
\hline 1 & 2.083 & 0.881 \\
\hline 2 & 4.167 & 1.762 \\
\hline 3 & 6.250 & 2.643 \\
\hline
\end{tabular}

\section{Image Analysis}

Taking the images at different exposures for each of these jet coefficients from 0 to 3 in increments of 0.5 , for four different models, resulted in hundreds of images for examination and comparison. Once fluorescent images are acquired, they are converted to .tiff images. For the images shown below, a picture of the model without any flow is added to the image for the model with the jet on at a given thrust coefficient. Qualitative images taken using the CCD camera system are shown below for both the parallel jet and transverse jet RCS configurations.

\section{B. LeMans Numerical Method (University of Michigan)}

Numerical simulations are performed using the computational fluid dynamics (CFD) code LeMANS, developed at the University of Michigan for simulating hypersonic reacting flows $[16,17]$. This general purpose, three-dimensional, parallel code solves the laminar Navier-Stokes equations on unstructured computational grids including thermo-chemical nonequilibrium effects with second-order accuracy. In LeMANS, the flow is modeled assuming that the continuum approximation is valid and that the fluid can be weakly ionized. The validity of the continuum approximation is best measured by Gradient-Length Local Knudsen number. Breakdown would occur for Knudsen values $>0.05$. Calculating this number for the experiment conditions, the continuum assumption is valid for the RCS jet region as shown in Figure 4. 


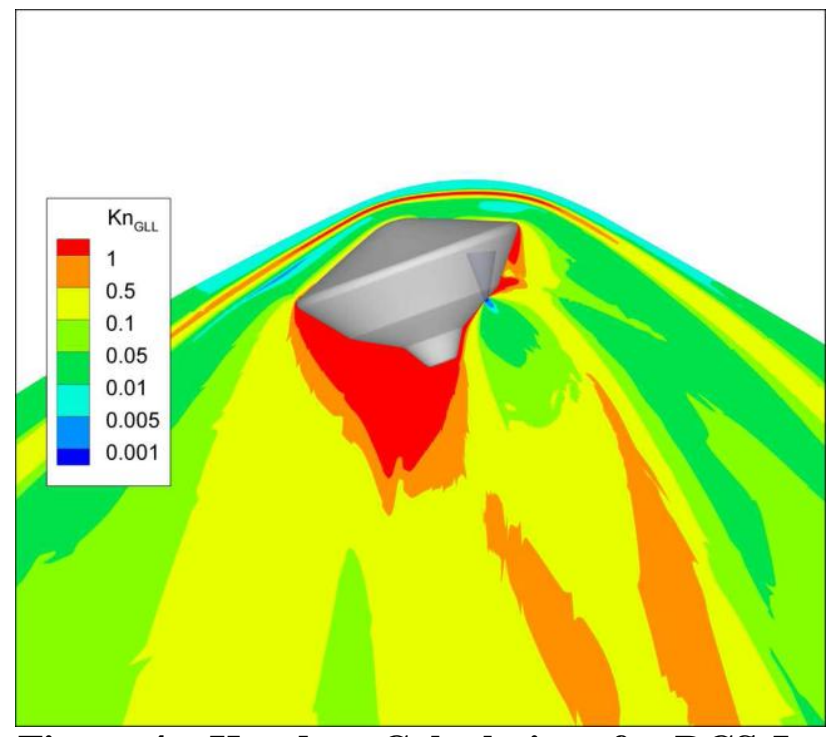

Figure 4 - Knudsen Calculations for RCS Jet

Furthermore, it is assumed that the translational and rotational energy modes of all species can be described by two different temperatures $T$ and $T_{r}$, respectively, while the vibrational energy mode and electron energy of all species can be described by a single temperature $T_{v}$ [18]. The electronic energy of atoms and molecules is neglected in the current version of the code due to the relatively small temperatures achieved in the hypersonic flows of interest. The mixture transport properties are computed using several options. In this study, Wilke's semi-empirical mixing is used with species viscosities calculated using Blottner's model and species thermal conductivities determined using Eucken's relation [19,20,21].

The finite-volume method applied to unstructured grids is used to solve the set of differential equations. LeMANS can simulate two-dimensional/axisymmetric flows using any mixture of quadrilateral and triangular mesh cells, and three-dimensional flows using any mixture of hexahedra, tetrahedra, prisms, and pyramids. It employs a modified Steger-Warming Flux Vector Splitting scheme to discretize the inviscid fluxes across cell faces, which is less dissipative and produces better results in boundary layers compared to the original scheme. Viscous terms are computed using cell center and node values. Time marching is performed using either a point implicit or a line implicit method. LeMANS is parallelized using METIS to partition the computational mesh between processors and MPI to communicate information between processors [22].

In order to better simulate the flow in the experimental facility, radially nonuniform conditions based on the supersonic freejet relations of Ashkenas and Sherman are used as boundary conditions to LeMANS. Fluid properties can be computed along the freejet axis using the Mach number defined by Ashkenas-Sherman given earlier, the stagnation conditions and the isentropic relations [10]. The density distribution as a function of the streamline angle $\theta$ with respect to the freejet axis is given by Eq. (3)

$$
\frac{\rho(\theta)}{\rho(0)}=\cos ^{2}\left(\frac{\pi \theta}{2 \Phi}\right)
$$


where $\Phi$ is also a constant based on the ratio of specific heats $\gamma$ and is equal to 1.662 for $\mathrm{N}_{2}$.

Although the $\mathrm{N}_{2}$ gas in the experimental facility is seeded with $\mathrm{I}_{2}$ with a seeding ratio of approximately $200 \mathrm{I}_{2}$ molecules per $10^{6} \mathrm{~N}_{2}$ molecules, Padilla showed that the differences in flowfield properties between $\mathrm{I}_{2}$-seeded and pure $\mathrm{N}_{2}$ gas are overall small, with slight discrepancies in velocity components in the boundary layer and the shock [23]. Therefore, pure $\mathrm{N}_{2}$ instead of $\mathrm{I}_{2}$-seeded gas is considered in the numerical simulations.

\section{Results and Discussion}

\section{A. Reaction Control System Jet Visualization}

Images shown in Figure 5 and 6 are given for nozzle-thrust coefficients from 0.5 to 3.0 in increments of 0.5 , focusing on the wind side of the RCS models. In each image, the model is oriented at a 20 degree angle of attack on its sting to the left of the image. The freestream flows from top to bottom, over the model, forming a bow shock from the aeroshell. The RCS jets, upon exiting the model at Mach 1, form a highly underexpanded jet with a well-defined barrel shock structure. Like with the underexpanded jet of the wind tunnel, this jet increases in Mach number reaching supersonic and hypersonic conditions before reaching a Mach disk. With an increase in thrust coefficient, the jet and its shock structure extend further beyond the model.

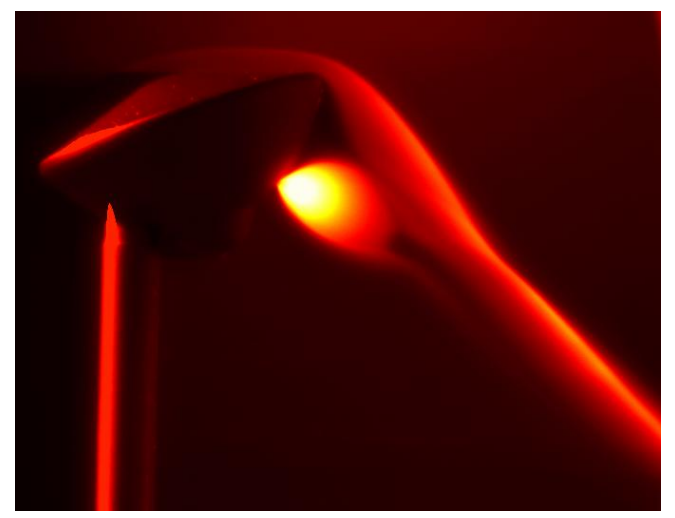

(a) $\mathrm{C}_{\mathrm{T}}=0.5$

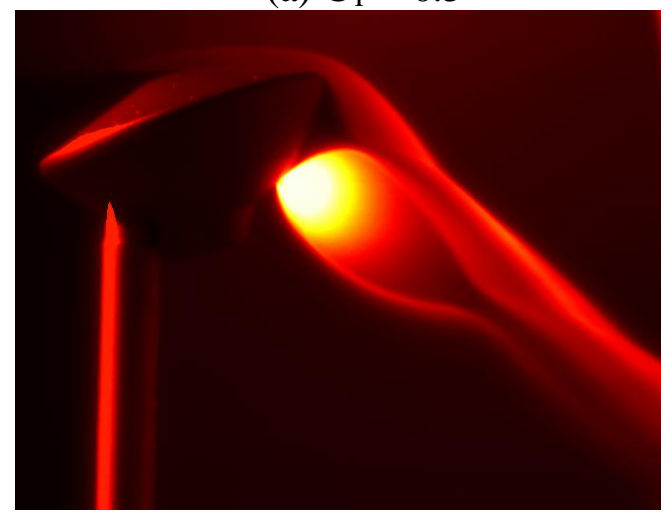

(c) $\mathrm{C}_{\mathrm{T}}=1.5$

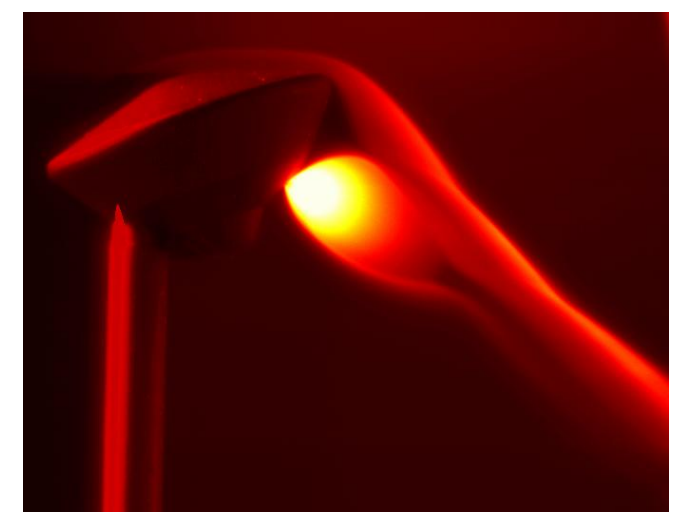

(b) $\mathrm{C}_{\mathrm{T}}=1.0$

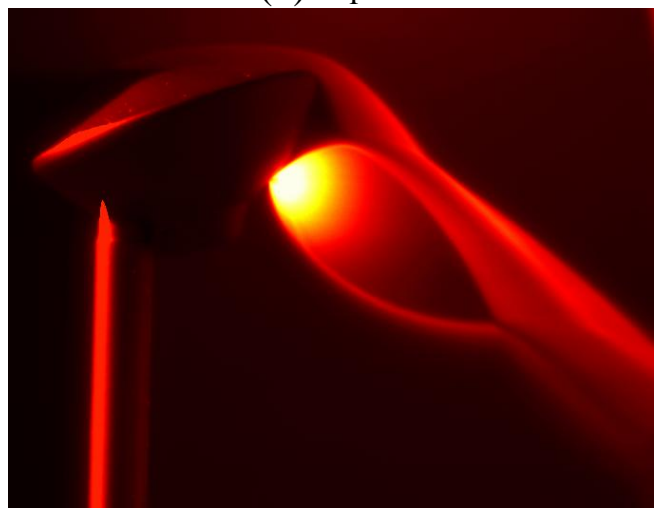

(d) $\mathrm{C}_{\mathrm{T}}=2.0$ 


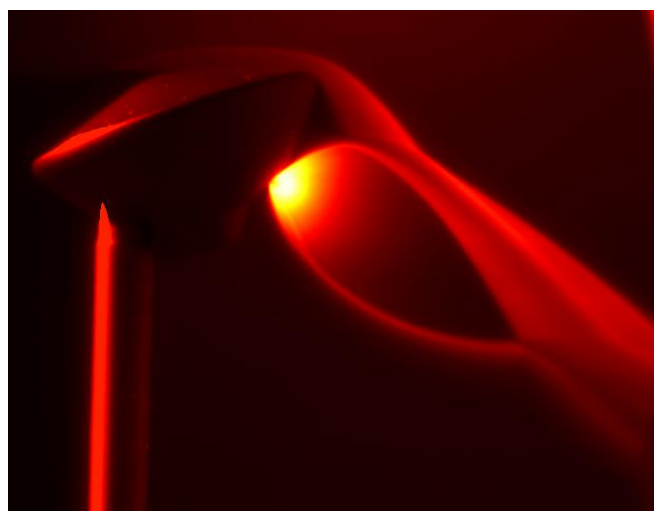

(e) $\mathrm{C}_{\mathrm{T}}=2.5$

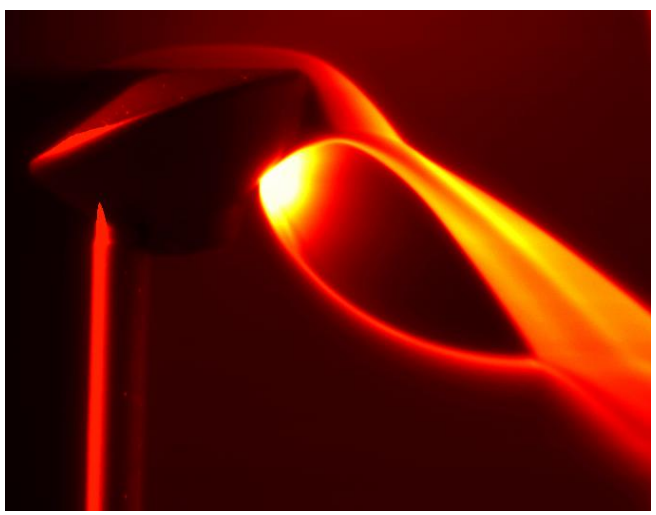

(f) $\mathrm{C}_{\mathrm{T}}=3.0$

Figure 5 - Transverse Jet Images

In Figure 5, there are a number of characteristics in the RCS transverse jet flow that become apparent. The first is the interaction of the aeroshell bow shock and the RCS jet barrel shock. As the nozzle-thrust coefficient increases, this interaction becomes more pronounced. Also, with increased thrust coefficient, the jet's Mach disk reduces in diameter until the barrel shocks merge. There is asymmetry in the RCS jet which exists at smaller thrust coefficients but is readily noticeable at higher thrust coefficient. This seems to be due to the interaction with the bow shock from the aeroshell. At higher nozzle-thrust coefficients, the aeroshell bow shock is pushed upstream and curves more tightly around the model.

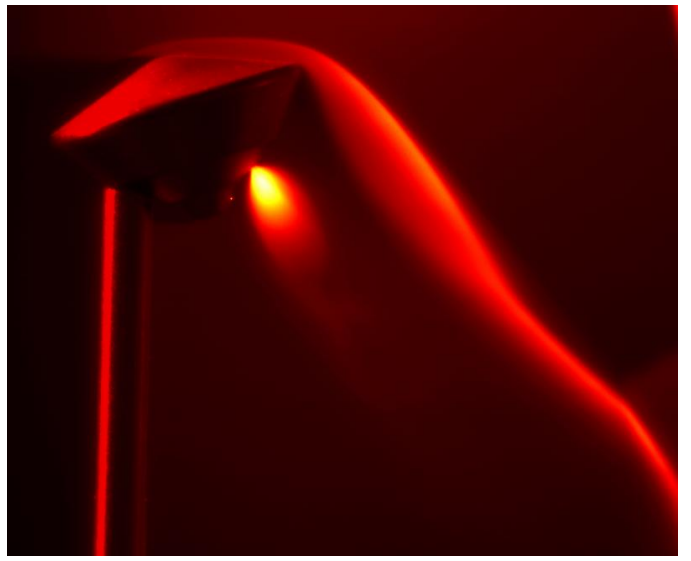

(a) $\mathrm{C}_{\mathrm{T}}=0.5$

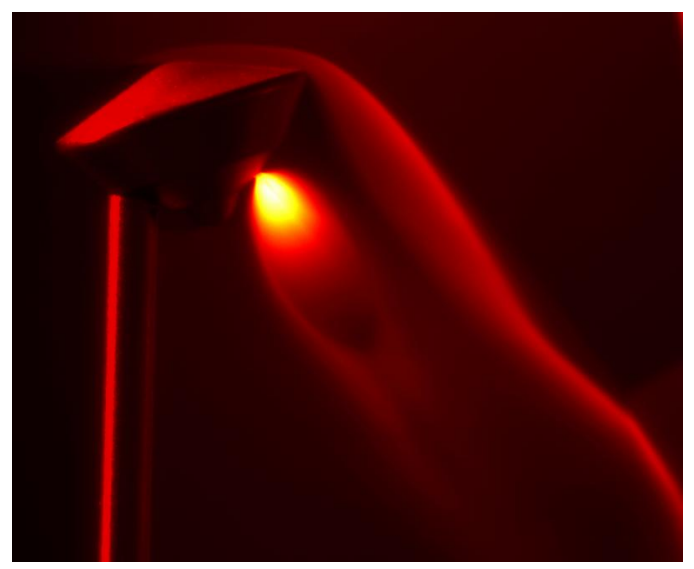

(b) $\mathrm{C}_{\mathrm{T}}=1.0$ 


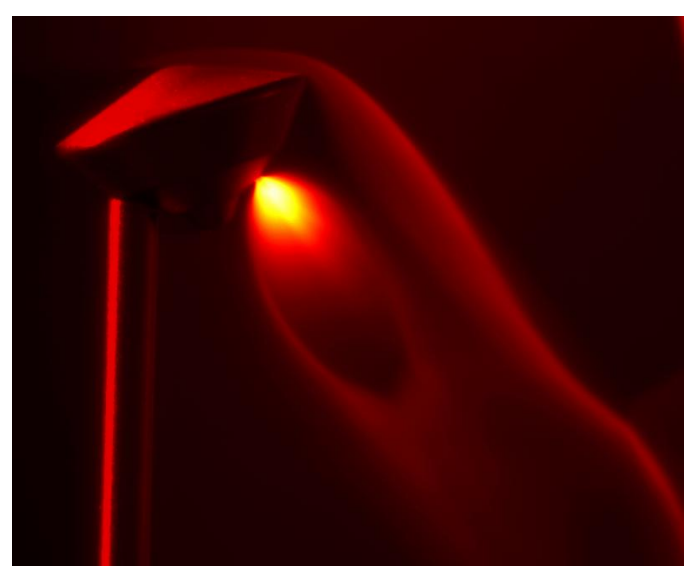

(c) $\mathrm{C}_{\mathrm{T}}=1.5$

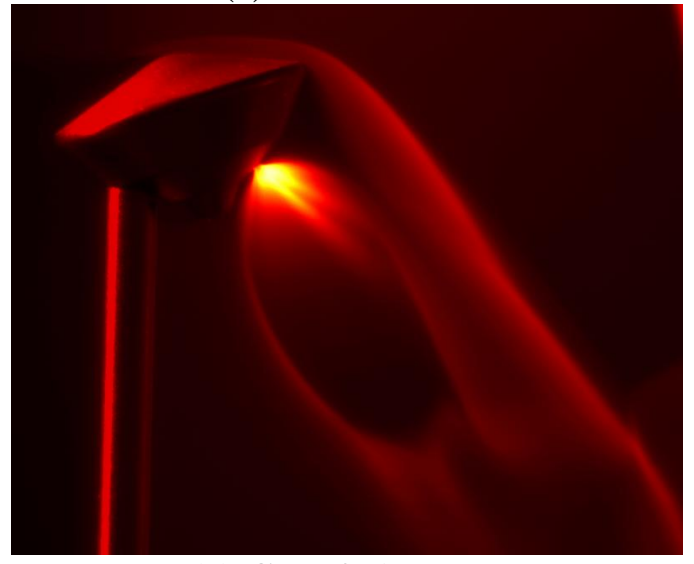

(e) $\mathrm{C}_{\mathrm{T}}=2.5$

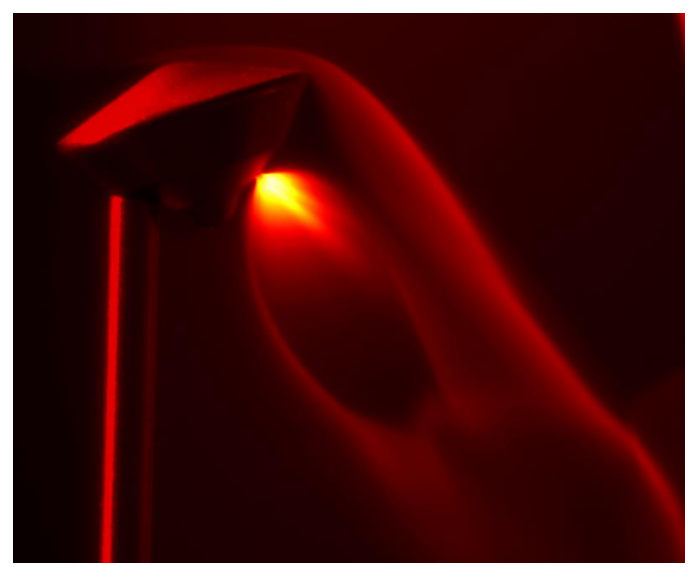

(d) $\mathrm{C}_{\mathrm{T}}=2.0$

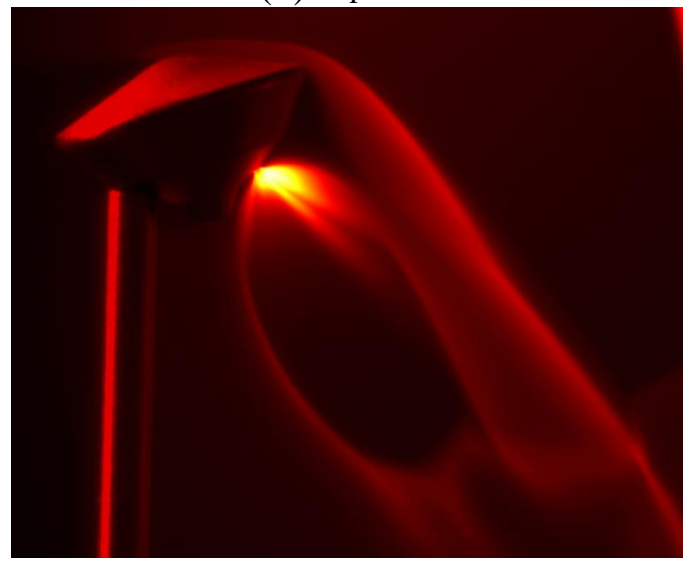

(f) $\mathrm{C}_{\mathrm{T}}=3.0$

\section{Figure 6 - Parallel Jet Images}

The parallel jet images (Figure 6), also show an asymmetry in the shape of the RCS jet. The shock structure for this jet is more clearly defined, including a pronounced Mach disk at higher nozzle-thrust coefficients. In comparing the bow shock shape throughout the series, there appears to be very little if any interaction between the aeroshell bow shock and the jet.

\section{B. Numerical Simulation}

Numerical simulations are performed using LeMANS assuming thermo-chemical equilibrium and the results are compared to the experimental data. Figure 7 shows Mach number contours on the plane of symmetry as predicted by LeMANS for the parallel RCS jet configuration with a nozzle thrust coefficient equal to 0.5 . The model used in the numerical simulations includes the converging section of the RCS nozzle to better match the experimental setup. The computational grid contains approximately 2.2 million hexahedral cells, with clustering near the MSL surface and RCS jet. Figure 7 illustrates the complex flow features and interactions that are generated during the use of these jets. The RCS jet expands from sonic conditions at the nozzle exit to supersonic and hypersonic conditions downstream in the wake region. The jet also disturbs most of the wake region on the windside, but does not seem to interact with the aeroshell bow shock. The Mach number distribution of the RCS jet is asymmetric in most of the wake region but eventually becomes symmetric at approximately 60 jet-exit diameters from the 
nozzle. This is the same trend seen in the PLIIF images in Figure 6. Sections A-A* and B-B* in Figure 7 are at 15 and 20 jet-exit diameters from the nozzle exit, respectively. They are used for comparisons between the numerical and experimental results.

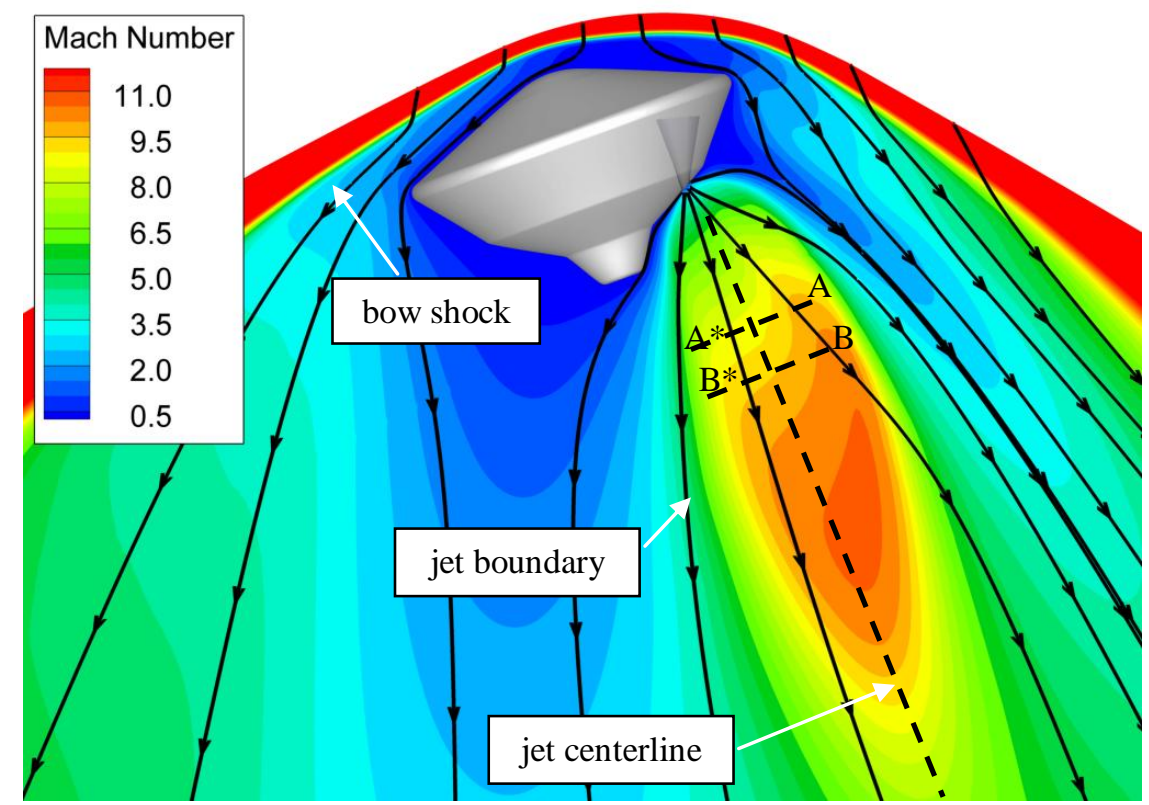

Figure 7 - RCS jet flowfield features.

\section{Experimental/Numerical Comparison}

Qualitative comparisons between the experimental and numerical results are also performed. The density distributions, normalized to jet exit conditions, along the jet centerline for the experimental measurements and numerical calculations are presented in Figure 8a. This figure shows that the experimental values are not proportional in a region near the nozzle exit (0-10 jet-exit diameters) because the normalized density values should not increase in an expansion. The reason for this discrepancy is that the diagnostic technique produces results that are not directly proportional to density in the continuum regions of the flowfield near the jet exit [14]. Between 10 and 25 jet-exit diameters away from the nozzle, the experimental and numerical results are in relatively good agreement, with a maximum difference of less than $10 \%$. Beyond 25 jet-exit diameters from the nozzle exit, the difference between the normalized density values for the two methods increases to about $10 \%$. The experimental measurements show that the normalized density values are almost constant, while LeMANS predicts that the density values continue to decrease.

Figure $8 \mathrm{~b}$ shows the normalized density values for the two methods across the RCS jet at 15 jet-exit diameters away from the nozzle exit (section A-A* in Figure 7). Near the jet core $(-4.5$ to +4.5 jet-exit diameters $)$, there is relatively good agreement between the two results, with a maximum difference of 14\%. Close to the jet boundary, however, there is a large disagreement between the two methods. LeMANS predicts higher normalized density values near the jet boundary compared to the experimental measurements. The reasons for this are that LeMANS predicts a larger RCS jet width at section A-A* than the experimental measurements and also the PLIIF signal is not proportional to density in the more continuum regions of the jet boundary. A second 
comparison can be seen in Figure 8c, which shows the normalized density values for the numerical and experimental methods across the RCS jet at 20 jet-exit diameters away from the nozzle exit (section B-B* in Figure 7). The comparison again shows that the differences between the numerical and experimental results are relatively small near the jet core, but increase near the jet boundary.

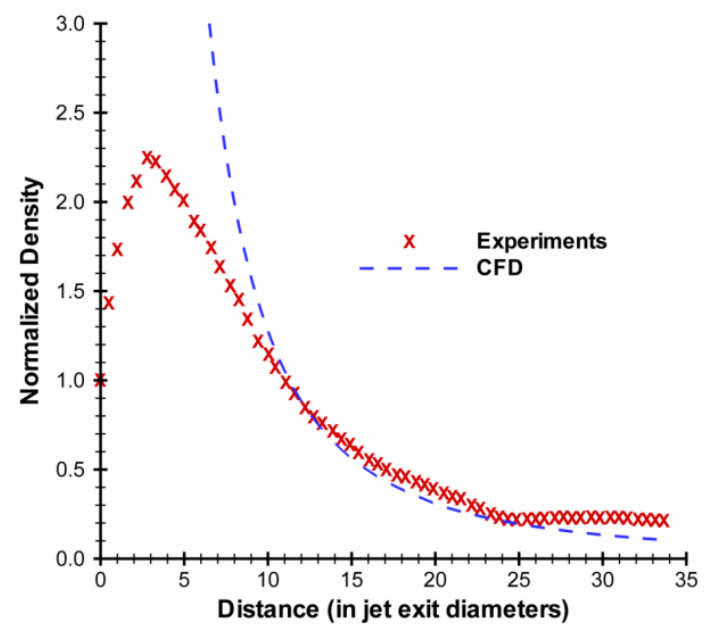

(a) jet centerline

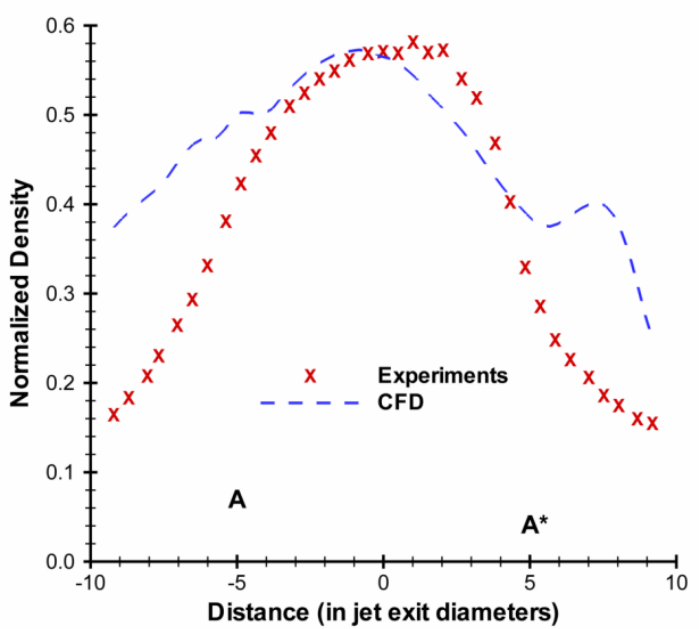

(b) 15 jet exit diameters (Section A-A*)

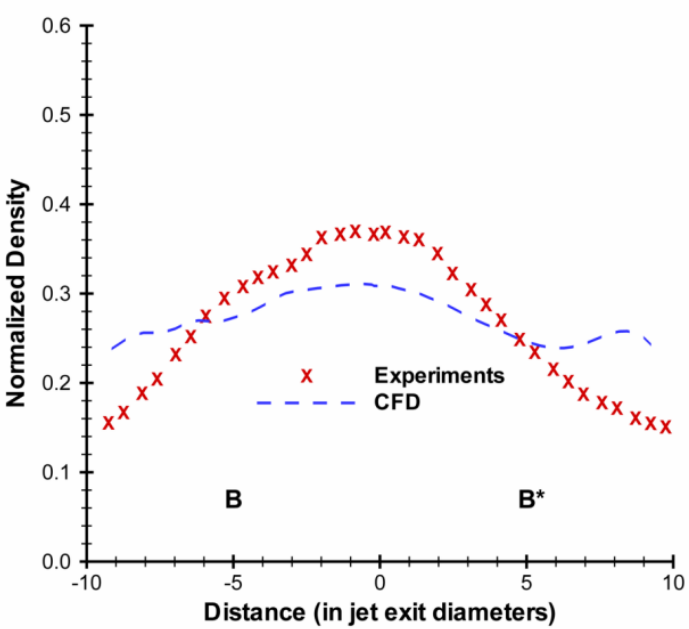

(c) 20 jet exit diameters (Section B-B*)

Figure 8 - Normalized density distributions for experimental and numerical results: (a) along the jet centerline (b) across the jet at 15 jet-exit diameters (c) across the jet at 20 jet-exit diameters.

Figures $9 \mathrm{a}$ and $9 \mathrm{~b}$ show density contours on the plane of symmetry from the experimental and numerical methods, respectively. In Figure 9a, the image on the left shows luminosity contours which are proportional to the density values in the rarefied regions of the flowfield, while the image on the right shows the same results at a different contrast ratio to highlight the density distribution and the fluid structure of the RCS jet. The contour levels in Figure $9 \mathrm{~b}$ for the numerical results are calibrated using the density values from the comparisons shown in Figure 8. Figure 9 shows overall good agreement 
between the two results in regards to the expansion of the jet along the centerline. The figure also shows that LeMANS, however, predicts an RCS jet width larger than observed in the experiments and a region of higher density values on the $\mathrm{A}^{*}$ - $\mathrm{B}^{*}$ sides of the jet centerline, which was also seen in Figure 8. These differences in the RCS jet profile between the numerical and experimental methods may be caused by slight differences in the geometry of the MSL model and the RCS nozzle used by the two approaches.
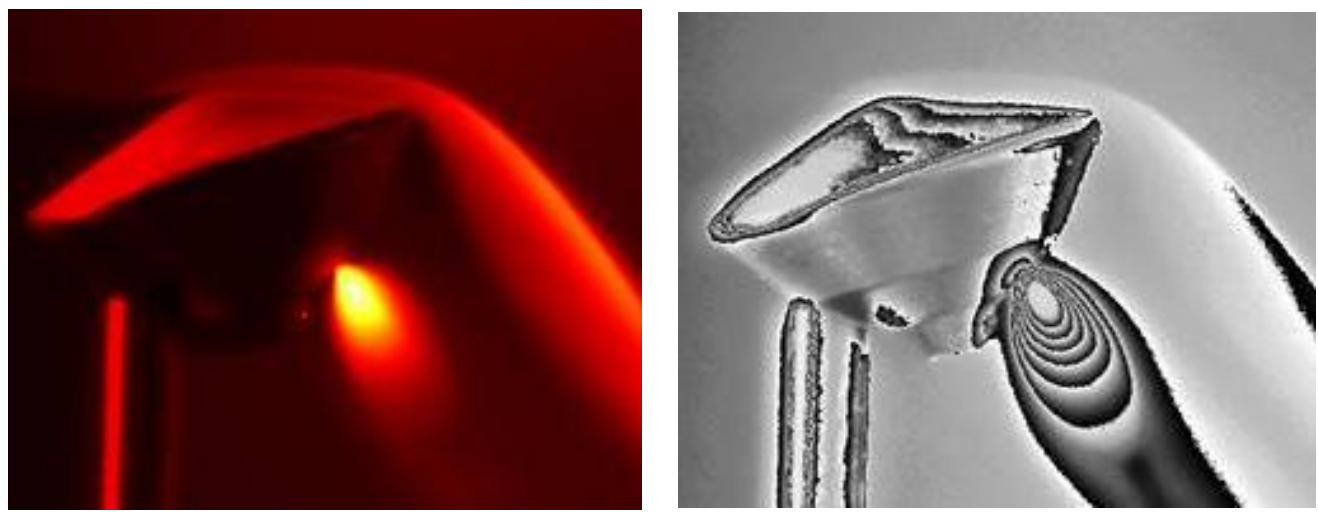

(a) experimental results
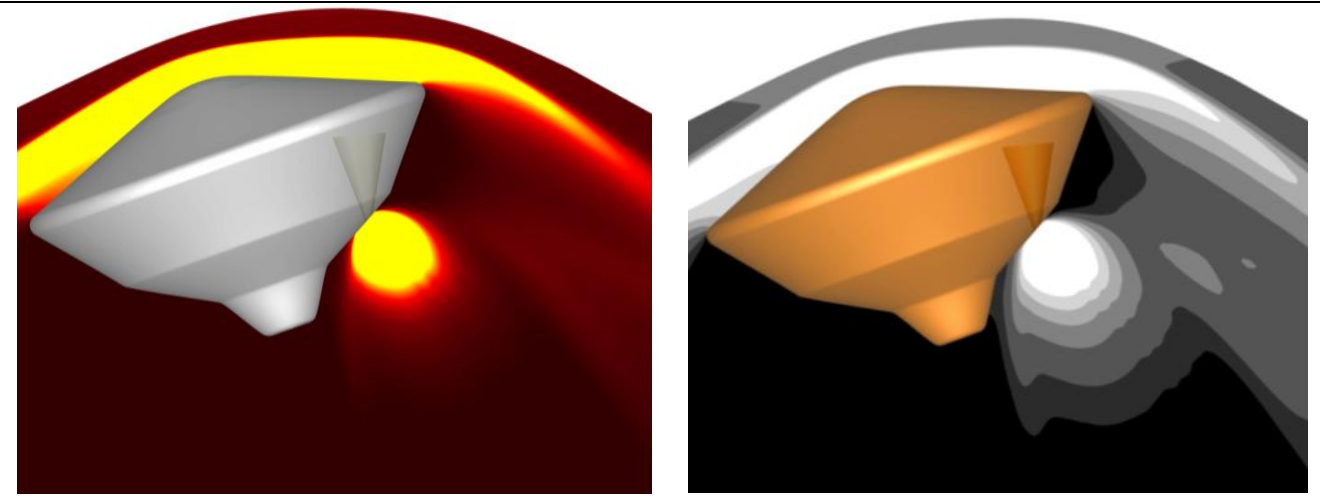

(b) numerical results

Figure 9 - Flowfield density distribution for: (a) experimental results (b) numerical results

\section{Conclusions}

Using Planar Laser Induced Iodine Fluorescence in a low density, hypersonic wind tunnel at the University of Virginia, images of MSL models fitted with Reaction Control System jets are obtained for nozzle-thrust coefficients of 0.5 to 3.0. These images show both transverse and parallel RCS jet interactions with the bow shock generated by the aeroshell. Results for parallel RCS jet at a nozzle-thrust coefficient of 0.5 are compared to a numerical simulation completed at the University of Michigan. Good agreement is found between these results in the RCS jet core but deviate at the jet boundaries. For future work, the internal shape of the RCS jets will be used in the numerical simulations to ensure identical geometries between the two approaches. Also, a grid convergence study will be conducted to guarantee grid-independent numerical 
results. Future experimental work will include quantitative spatially-resolved measurements of velocity and temperature that can be used for further comparison to CFD.

\section{Acknowledgements}

This research was supported by NASA Grant NNX08AH37A and NIA/NASA grant VA03-01-2650 as well as a National Science Foundation Graduate Research Fellowship (Reed).

\section{References}

1. Braun, R.D., and Manning, R.M., "Mars Exploration Entry, Descent and Landing Challenges". IEEE Paper \#0076(2006).

2. Rivellini,T. National Academy of Engineering Website. National Academy of Engineering Website. [Online] 2004. [Cited: April 30, 2009.] http://www.nae.edu/NAE/bridgecom.nsf/BridgePrintView/MKEZ-69....

3. Lockwood, M.K., "Introduction: Mars Science Laboratory: The Next Generation of Mars Landers". Journal of Spacecraft and Rockets. Vol 43, No. 2 p.257 (2006).

4. Steltzner A., Kipp, D., Chen, A., Burkhart, D., Guernsey, C., Mendeck, G., Micheltree, R., Powell, R., Rivellini, T., San Martin, M., and Way, D., "Mars Science Laboratory Entry, Descent, and Landing System”. IEEE Paper \#1497 (2006)

5. Mitcheltree, R., Steltzner, A., Chen, A., San Martin, M., and Rivellini,T., "Mars Science Laboratory Entry Descent and Landing System Verification and Validation Program". IEEE Paper \#1498 (2006).

6. Way, D.W., Powell, R.W., Chen, A., Steltzner, A.D., San Martin, A.M., Burkhart, P.D., Mendeck, G.D., "Mars Science Laboratory: Entry, Descent, and Landing System Performance". IEEE Paper \#1467 (2006).

7. Braun, R.D., "Importance of Simulation". Planetary Entry, Descent and Landing - Hypersonic Educational Initiative. Hampton, VA : National Institute of Aerospace, April 1, 2008.

8. Danehy, P.M., Inman, J.A., Brauckmann, G.J, Alderfer, D.W., Jones, S.B. and Patry, D.P., "Visualization of a Capsule Entry Vehicle Reaction-Control System Thruster" Journal of Spacecraft and Rockets, vol. 46 no.1 p. 93-102 (2009).

9. Staack, D. and Del Vecchio, T.D., "The Design Building and Calibration of a Hypersonic Wind Tunnel" AIAA Paper 2000-0004, 2000.

10. Ashkenas, H. and Sherman, F.S., "The Structure and Utilization of Supersonic Free Jets in Low Density Wind Tunnels”. New York: Academic Press, 1966. Rarefied Gas Dynamics. pp. 84 $-105$.

11. Cecil, D.E. "Planar Laser-Induced Iodine Fluorescence Measurements in Rarefied Hypersonic Flow Over a Reaction Control Jet Model in a Free Jet Wind Tunnel". M.S. Thesis.

Charlottesville, VA : University of Virginia, January 2004.

12. Cecil, D.E. and McDaniel, J.C., "Planar Laser-Induced Iodine Fluorescence Measurements in Rarefied Hypersonic Flow". Toronto, Canada : American Institute of Physics, 2005. Rarefied Gas Dynamics: 24th International Symposium. pp. 1325-1350.

13. Hartfield R.J. Hollo, S.D. and McDaniel, J.C., "Planar Temperature Measurement in Compressible Flows Using Laser-Induced Iodine Fluorescence".Optics Letters, 1991, Vol. 16. 14. Staack, D. et al. "Experimental Study of Interacting Rarefied and Continuum Flows". AIAA Paper 2001-2762, 2001. 
15. Korzun, A.M., Cruz, J.R. and Braun, R.D., "Survey of Supersonic Retropropulsion Technology for Mars Entry, Descent, and Landing". Journal of Spacecraft and Rockets. Vol. 46, no. 5, pp 929 (2009).

16. Scalabrin, L.C. and Boyd, I.D., "Development of an Unstructured Navier-Stokes Solver for Hypersonic Nonequilibrium Aerothermodynamics", AIAA Paper 2005-5203, June 2005.

17. Scalabrin, L.D. and Boyd, I.D., "Numerical Simulation of Weakly Ionized Hypersonic Flow for Reentry Configurations", AIAA Paper 2006-3773, June 2006.

18. Holman, T.D. and Boyd, I.D., "Numerical Investigation of the Effects of Continuum Breakdown on Hypersonic Vehicle Surface Properties", AIAA Paper 2008-3928, June 2008.

19. C.R. Wilke, "A Viscosity Equation for Gas Mixtures", Journal of Chemical Physics, Vol. 18, 1950, pp. 517-519.

20. Blottner, F. G., Johnson, M., and M. Ellis, "Chemically Reacting Viscous Flow Program for Multi-Component Gas Mixtures”, Sandia Laboratories Report SC-RR-70-754, 1971.

21. Vincenti, W. G., and Kruger, C. H., Introduction to Physical Gas Dynamics, Krieger Publishing Company, 2002.

22. Karypis, G., and Kumar, V., METIS: A Software Package for Partitioning Unstructured Graphs, Partitioning Meshes, and Computing Fill-Reducing Orderings of Sparse Matrices, University of Minnesota, 1998.

23. Padilla, J. F., "Assessment of Gas-Surface Interaction Models for Computation of Rarefied Hypersonic Flows", Ph.D. Thesis, Department of Aerospace Engineering, University of Michigan, Ann Arbor, MI, 2008. 